\title{
Aortic stiffness is associated with the central retinal arteriolar equivalent and retinal vascular fractal dimension in a population along the southeastern coast of China
}

\author{
Fan Lin ${ }^{1}$, Pengli Zhu ${ }^{1}$, Feng Huang ${ }^{1}$, Qiaowei $\mathrm{Li}^{1}$, Yin Yuan ${ }^{1}$, Zhonghai Gao ${ }^{2}$, Peng $\mathrm{Yu}^{1}$, \\ Jing Lin $^{1}$ and Falin Chen ${ }^{3}$
}

The objective of this study was to evaluate the association of the central retinal arteriolar equivalent (CRAE) and the retinal vascular fractal dimension, two quantitative parameters that reflect microcirculation, with aortic stiffness. In this cross-sectional study, we identified the cardiovascular risk factors in 2169 subjects using a health questionnaire, physical examinations and laboratory examinations. We evaluated the aortic stiffness using noninvasive brachial-ankle pulse wave velocity (baPWV) and assessed the microcirculatory alterations with CRAE and retinal vascular fractal dimension, which were measured using fundus photography and semiautomatic quantitative software, respectively. The increase in baPWV (Q1-Q4) correlated with an increased likelihood of the central retinal artery narrowing and a reduction in the retinal vascular fractal dimension. Further adjustment of the cardiovascular risk factors diminished the association between baPWV and CRAE, but increased the association between baPWV and retinal vascular fractal dimension. Elevated baPWV correlates with reduced CRAE and retinal vascular fractal dimension. Such a finding supports macrocirculation- and microcirculation-associated hypotheses.

Hypertension Research (2015) 38, 342-348 doi:10.1038/hr.2015.11; published online 26 February 2015

Keywords: aortic stiffness; central retinal arteriolar; fractal dimension

\section{INTRODUCTION}

Recent advances in software development have enabled the quantitative analysis of the retinal blood vessels for cardiovascular disease research. The central retinal arteriolar equivalent (CRAE) has been shown to be associated with many cardiovascular risk factors. ${ }^{1-6}$ Furthermore, it was found that a narrow central retinal artery correlated with coronary heart disease and hypertension and was independently predictive of cardiovascular diseases and peripheral arterial embolism that develop within 6 years. The human circulatory system operates on an optimal structural design, enabling adequate blood flow with minimal energy consumption. Therefore, a suboptimal microcirculation is considered harmful to the vascular integrity. ${ }^{7}$ The retinal vascular fractal dimension is a mathematical indicator measuring the complexity of the retinal blood vessels. It has been shown that the retinal vascular fractal dimension is associated with mortality in hypertension, ${ }^{8,9}$ diabetic retinopathy, ${ }^{10,11}$ chronic kidney disease, ${ }^{12}$ stroke ${ }^{13-15}$ and coronary heart diseases. ${ }^{16}$
Early signs of atherosclerosis are associated with the loss of elasticity in large- and medium-sized arteries. ${ }^{17,18}$ This increased arterial stiffness correlates with a series of cardiovascular risk factors, in addition to its association with age. ${ }^{19-21}$ Indeed, increased aortic stiffness has proven to be an independent risk factor in cardiovascular illness and all-cause mortality. ${ }^{22-26}$ Noninvasive brachial-ankle pulse wave velocity (baPWV) has been widely used to clinically determine aortic stiffness. Owing to the relatively easier measurement of the carotid-femoral pulse wave velocity, baPWV has been used in screening for vascular damage and cardiovascular risk assessment.

The correlation between arterial stiffness and microvascular diseases has been described. ${ }^{27-30}$ However, the association of microvascular complexity and microcirculatory damage as a measure of retinal vascular fractal dimension in aortic stiffness has yet to be elucidated. Therefore, to evaluate the correlation between aortic stiffness and microvascular lesions, we designed this cross-sectional study to investigate the association of CRAE and retinal vascular fractal dimension with aortic stiffness in a Chinese population.

${ }^{1}$ Department of Geriatric Medicine, Fujian Provincial Hospital, Fujian Provincial Institute of Clinical Geriatrics, Provincial Clinical Medical College of Fujian Medical University, Fuzhou, China; ${ }^{2}$ Department of Ophthalmology, Fujian Provincial Hospital, Fuzhou, China and ${ }^{3}$ Clinical Laboratory Center, Fujian Provincial Hospital, Fuzhou, China Correspondence: Professor P Zhu, Department of Geriatric Medicine, Fujian Provincial Hospital, Fujian Provincial Institute of Clinical Geriatrics, Provincial Clinical Medical College of Fujian Medical University, Fuzhou 350001, China.

E-mail: zp17755@gmail.com

Received 9 May 2014; revised 29 September 2014; accepted 31 October 2014; published online 26 February 2015 


\section{METHODS}

Subjects

Cluster sampling was performed from July 2011 through November 2011. The clusters were the individual administrative villages, and we sought to obtain 7 sampling units in 14 villages for two specified townships. The villages of Tailu, Beijiao and Xiubang in the Tailu township of Lienchiang county and the villages of Kungtung, Kunghsi, Yantai and Wenwo in the Haidao township of Xiapu county in Fujian Province were randomly selected for this cross-sectional investigation. Invitations to participate in the survey were sent to 4616 subjects who were sampled from the 8947 inhabitants, aged 30 years and above and registered in local areas based on the age stratification. A total of 3343 subjects participated in the survey. We excluded 1174 subjects from the analysis because of incomplete data (421 subjects), affliction with infectious disease (C-reactive protein level $>10 \mathrm{mgl}^{-1} ; 49$ subjects) or atrial fibrillation (14 subjects), low ankle brachial index $(<0.6 ; 15$ subjects) and unqualified or unclear fundus photographs that affected the analysis (675 subjects). In the final analysis, only 2169 subjects were involved. This study was approved by the ethics committee of the Fujian Provincial Hospital, China. Informed consent was obtained from all participants following a detailed description of the potential benefits and risks associated with the study.

\section{Data collection}

The age, smoking habits, alcohol consumption, medical history (including hypertension, diabetes, coronary heart disease, stroke, liver and renal dysfunction, malignant tumors and peripheral vascular diseases) and family history were investigated using a questionnaire.

The blood pressure was measured with a standard vertical mercury sphygmomanometer. Before the measurement, the subjects were placed in a sitting position and allowed to rest quietly for more than $10 \mathrm{~min}$. Then, the systolic and diastolic blood pressure of the right upper arm was measured. Blood pressure was measured three times, $5 \mathrm{~min}$ apart, and the mean blood pressure was estimated in triplicate.

Blood samples were collected from the subjects in the morning after $8 \mathrm{~h}$ of fasting to determine the plasma levels of triglyceride, total cholesterol, highdensity lipoprotein cholesterol (HDL-C), low-density lipoprotein cholesterol (LDL-C), fasting glucose, glycosylated hemoglobin $\left(\mathrm{HbA}_{1 \mathrm{c}}\right)$, hypersensitive C-reactive protein (hs-CRP) and serum uric acid (SUA).

\section{Measurement of baPWV}

The baPWV was measured by two competent and well-trained professionals, after the subjects rested quietly for $10 \mathrm{~min}$. The arterial pulse waveform was recorded for $5 \mathrm{~min}$ using a fully automatic arteriosclerosis detector (Colin VP-1000 device; Colin Medical Technology Company, Komaki, Japan), displaying the baPWV automatically. The right baPWV was selected for data analyses.

\section{Fundus photography and quantitative analysis of the retinal vascular parameters}

High-resolution fundus photography using a digital non-mydriatic camera was performed on both eyes (Topcon NW-8, TOPCON CORPORATION, Tokyo, Japan and Nikon D90, Tokyo, Japan) with a capturing range of $45^{\circ}$ using the optic disk as the center (Figures 1 and 2). A double-blind analysis of the photographs was performed by two professionally trained ophthalmologists, and high-quality fundus photographs were used for analysis. We used a semiautomated computer-based program (Singapore I Vessel Assessment version 3.0 software, jointly developed by Singapore National University and Singapore Eye Research Institute, Singapore) for the quantitative analysis. The CRAE was measured using the modified Knudtson-Parr-Hubbard formula ${ }^{31}$ in the range of $0.5-1$ DD from the disc margin, and the retinal vascular fractal dimension was measured in the range of 0.5-2 DD from the disc margin.

\section{Definitions}

Hypertension was defined as the systolic blood pressure of $\geqslant 140 \mathrm{~mm} \mathrm{Hg}$ and/ or the diastolic blood pressure of $\geqslant 90 \mathrm{~mm} \mathrm{Hg}$ in the absence of

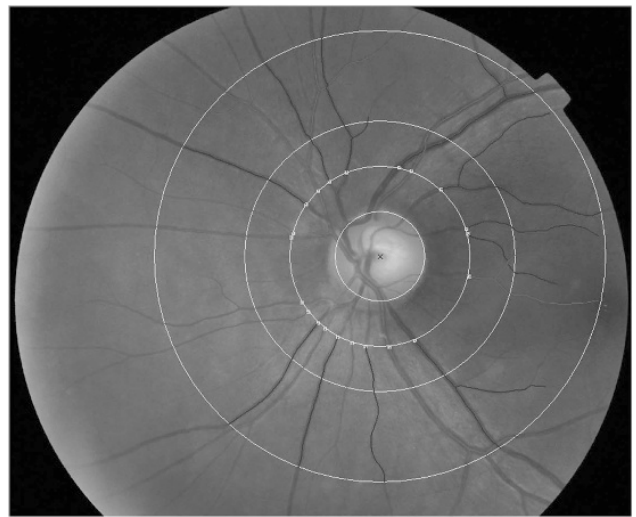

Figure 1 Retinal fundus photograph quantitatively assessed using the Singapore I Vessel Assessment version 3.0 software. Arterioles are in red, and venules are in blue. The measured area of the retinal vascular quantitative parameters were standardized and defined as the region from 0.5 to 2.0 disc diameters away from the disc margin. The full colour version of this figure is available at Hypertension Research online.

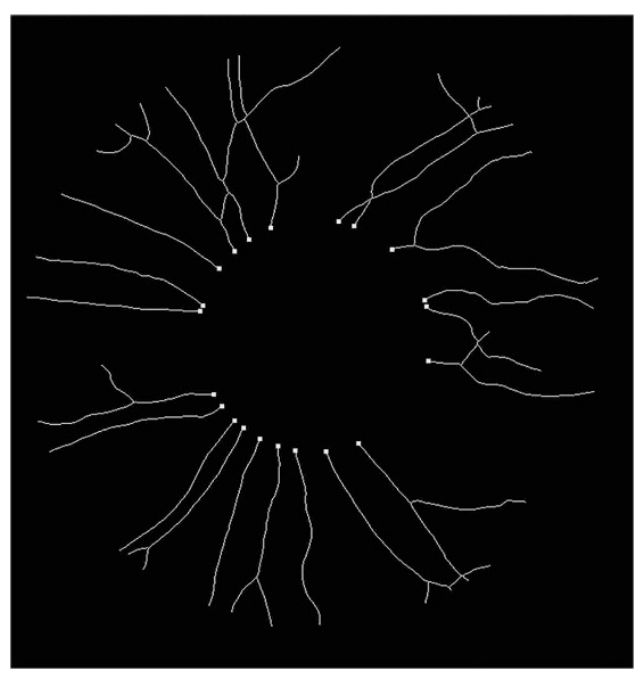

Figure 2 Retinal fundus photograph quantitatively assessed using the Singapore I Vessel Assessment version 3.0 software. The retinal vascular fractal dimension was calculated from the skeletonized line tracing using the box-counting method. The full colour version of this figure is available at Hypertension Research online.

antihypertensive drug therapy. The subjects with a history of hypertension and who were currently using antihypertensive drugs were also defined as hypertensive. $^{32}$

Diabetes was defined as an $\mathrm{HbA}_{\mathrm{lc}}$ of $\geqslant 6.5 \%$. Additionally, patients with a history of diabetes, or who were currently using antidiabetic drugs were considered diabetic. ${ }^{33}$

The cutoff value for the normal and increased baPWV was $1400 \mathrm{~cm} \mathrm{~s}^{-1} .34$ The lowest quartile of CRAE was defined as the central retinal artery narrowing. ${ }^{35}$ Similarly, the lowest quartile of the retinal vascular fractal dimension was defined as retinal vascular fractal dimension decreasing. The mean arterial blood pressure, the pulse pressure and the body mass index (BMI) were calculated using the following formulae: mean arterial blood pressure $(\mathrm{mm} \mathrm{Hg})=1 / 3$ systolic blood pressure $+2 / 3$ diastolic blood pressure; pulse pressure $(\mathrm{mm} \mathrm{Hg})=$ systolic blood pressure - diastolic blood pressure; BMI $\left(\mathrm{kg} \mathrm{m}^{-2}\right)=$ body weight per height ${ }^{2}$. The mean arterial blood pressure and the pulse pressure were grouped according to the quartile (Q1-Q4); BMI was grouped into normality or malnutrition $\left(<25 \mathrm{~kg} \mathrm{~m}^{-2}\right)$, overweight or obesity $\left(\geqslant 25 \mathrm{~kg} \mathrm{~m}^{-2}\right)$; HbAlc levels were assigned to the normal HbAlc group $(<6.5 \%)$ 
and abnormal $\mathrm{HbAlc}$ group $(\geqslant 6.5 \%)$; and the LDL-C levels were assigned into the normal LDL group $\left(<3.3 \mathrm{mmol}^{-1}\right)$ and abnormal LDL-C group $(\geqslant 3.3$ mmol $\left.1^{-1}\right)$. According to the Third Report of the Expert Panel on Detection, Evaluation and Treatment of High Blood Cholesterol in Adults (Adult Treatment Panel III) of the National Cholesterol Education Program, the serum lipid levels were grouped as the normal serum lipid group (LDL-C $<3.3 \mathrm{mmoll}^{-1}$, HDL-C $\geqslant 1.0 \mathrm{mmoll}^{-1}$, serum cholesterol level $<5.2 \mathrm{mmoll}^{-1}$ and triglyceride $<1.7 \mathrm{mmoll}^{-1}$ ) and the abnormal serum lipid group.

\section{Statistical analysis}

All statistical analyses were performed using the statistical software SPSS (Statistical Package for the Social Sciences) version 17.0.1 (SPSS Inc., Chicago, IL, USA), with a $P$-value $<0.05$ indicative of statistical significance.

After the baPWV stratification, the subjects' baseline characteristics were compared. The retinal vascular fractal dimension was normally distributed. Other variables were obtained from a large number of random and independent observations such that the arithmetic average of the observed values was approximately normally distributed. The skewness of the CRP was 93.23, which was much $>1.96$ times its standard error; hence, the CRP was considered right-skewed distributed. The skewed distributed variables were taken as approximately normal distributions after a logarithmic transformation was performed. The normally distributed or the approximately normally distributed data were expressed as the mean \pm s.d., and the data with skewed distribution were described as the median (upper and lower quartiles). The count data were expressed as proportions.

The associations of the gender- and the age-adjusted baPWV, CRAE and the retinal vascular fractal dimension with the stratified cardiovascular risk factors were evaluated using analysis of covariance, and the stratified cardiovascular risk factors were used as continuous variables for the trend test. The changes in the retinal vascular parameters (CRAE and the retinal vascular fractal dimension) associated with the increase in baPWV (Q1-Q4) were assessed using analysis of covariance, with the stratified baPWVs used as continuous variables for the trend test. All data in the model were age- and genderadjusted. The probability of changes in the retinal vascular parameters of the lower quartile group with the increase in baPWV (Q1-Q4) was investigated using a multivariate logistic regression, and two models were therefore designed. In the first model, the variables including gender, age, smoking habits and alcohol consumption were controlled. Conversely, in the second model, the variables including gender, age, smoking habits, alcohol consumption, diabetes, systolic blood pressure, BMI, abnormal lipid level, hs-CRP level and high SUA level were controlled.

\section{RESULTS}

The baseline characteristics of the 2169 subjects enrolled in this study and their grouping according to the baPWV are described in Table 1. The subjects enrolled in this study included 812 males and 1357 females, with a mean age $51.92 \pm 11.91$ years, a mean baPWV of $1417.72 \pm 338.07 \mathrm{~cm} \mathrm{~s}^{-1}$, a mean CRAE of $134.10 \pm 11.13 \mu \mathrm{m}$ and a mean retinal vascular fractal dimension of $1.37 \pm 0.05$. The subjects were assigned to the normal group and the arteriosclerosis group according to their baPWV levels. The mean age, systolic blood pressure, diastolic blood pressure, arterial blood pressure, pulse pressure, BMI, triglyceride, total cholesterol, LDL-C, plasma fibrinogen, HbAlc, hs-CRP and SUA in the arteriosclerosis group were higher than those in the normal group, whereas the mean HDL-C level in the arteriosclerosis group was lower than that in the normal group. In addition, the percentage of male subjects, smoking habits, alcohol consumption and the prevalence of diabetes in the arteriosclerosis group were greater than those in the normal group. The mean CRAE $(132.68 \pm 11.73 \mu \mathrm{m})$ and the mean retinal vascular fractal dimension $(1.35 \pm 0.05)$ in the arteriosclerosis group were lower than those in the normal group $(135.06 \pm 10.61 \mu \mathrm{m}$ and $1.89 \pm 0.05)$.

Table 1 Characteristics of study population (baPWV status)

\begin{tabular}{|c|c|c|c|}
\hline Characteristics & All & Normal (baPWV <1400 $\mathrm{cm} \mathrm{s}^{-1}$ ) & Increased baPWV (baPWV $\left.\geqslant 1400 \mathrm{~cm} \mathrm{~s}^{-1}\right)$ \\
\hline Subjects $(n)$ & 2169 & 1296 & 873 \\
\hline Age (years) & $51.92 \pm 11.91$ & $46.54 \pm 9.52$ & $59.91 \pm 10.54$ \\
\hline $\mathrm{SBP}(\mathrm{mm} \mathrm{Hg})$ & $126.94 \pm 21.94$ & $116.16 \pm 14.68$ & $142.94 \pm 21.15$ \\
\hline DBP $(m m ~ H g)$ & $78.55 \pm 11.85$ & $74.50 \pm 10.05$ & $84.58 \pm 11.77$ \\
\hline MABP $(\mathrm{mm} \mathrm{Hg})$ & $94.67 \pm 14.10$ & $88.36 \pm 10.82$ & $104.04 \pm 13.17$ \\
\hline Pulse pressure $(\mathrm{mm} \mathrm{Hg})$ & $48.39 \pm 15.88$ & $41.66 \pm 10.13$ & $58.37 \pm 17.54$ \\
\hline $\mathrm{HR}$ (per min) & $71.22 \pm 9.28$ & $70.78 \pm 8.71$ & $71.87 \pm 10.03$ \\
\hline $\mathrm{BMI}\left(\mathrm{kg} \mathrm{m}^{-2}\right)$ & $23.83 \pm 3.42$ & $23.32 \pm 3.38$ & $24.58 \pm 3.33$ \\
\hline Triglyceride $\left(\mathrm{mmol} \mathrm{I}^{-1}\right)$ & $0.86 \pm 0.50$ & $0.92 \pm 0.64$ & $0.76 \pm 0.01$ \\
\hline Total cholesterol (mmol $\left.{ }^{-1}\right)$ & $5.03 \pm 1.05$ & $4.88 \pm 1.02$ & $5.24 \pm 1.06$ \\
\hline $\mathrm{HDL}-\mathrm{C}\left(\mathrm{mmol} \mathrm{I}^{-1}\right)$ & $1.23 \pm 0.33$ & $1.23 \pm 0.32$ & $1.22 \pm 0.34$ \\
\hline LDL-C (mmol// $\left.{ }^{-1}\right)$ & $2.79 \pm 0.88$ & $2.65 \pm 0.82$ & $2.99 \pm 0.94$ \\
\hline FPG (mmoll) & $5.31 \pm 1.50$ & $5.17 \pm 1.10$ & $5.53 \pm 1.93$ \\
\hline HbAlc (\%) & $5.72 \pm 0.68$ & $5.59 \pm 0.49$ & $5.90 \pm 0.87$ \\
\hline hs-CRP (mg |-1) & $0.52(0.31,1.34)$ & $0.38(0.25,1.00)$ & $0.79(0.48,1.93)$ \\
\hline SUA $(\mu \mathrm{mol} \mathrm{I}-1)$ & $315.04 \pm 84.82$ & $306.98 \pm 83.84$ & $327.00 \pm 84.91$ \\
\hline baPWV $\left(\mathrm{cm} \mathrm{s}^{-1}\right)$ & $1417.72 \pm 338.07$ & $1202.81 \pm 120.12$ & $1736.76 \pm 303.60$ \\
\hline CRAE $(\mu \mathrm{m})$ & $134.10 \pm 11.13$ & $135.06 \pm 10.61$ & $132.68 \pm 11.73$ \\
\hline Retinal vascular fractal dimension & $1.37 \pm 0.05$ & $1.89 \pm 0.05$ & $1.35 \pm 0.05$ \\
\hline Gender (\%male) & $812(37.4)$ & $419(32.3)$ & $393(45.0)$ \\
\hline Cigarette smoking (\%) & $415(19.1)$ & $213(16.4)$ & $202(23.1)$ \\
\hline Alcohol drinking (\%) & $341(15.7)$ & $164(12.7)$ & $176(20.2)$ \\
\hline Diabetes (\%) & $272(12.5)$ & 99 (7.6) & $173(19.8)$ \\
\hline
\end{tabular}

Abbreviations: baPWV, brachial-ankle pulse wave velocity; $\mathrm{BMI}$, body mass index. $\mathrm{BP}$, systolic blood pressure; $\mathrm{Cl}$, confidence interval; CRAE, central retinal arteriolar equivalent. DBP, diastolic blood pressure. FPG, fasting plasma glucose; HbAlc, hypersensitive; HDL-C, high-density lipoprotein cholesterol; HR, heart rate; hs-CRP, hypersensitive C-reactive protein; LDL-C, low-density lipoprotein cholesterol; MABP, mean arterial blood pressure; SUA, serum uric acid; TC, total cholesterol; TG, triglyceride.

Homogeneous continuous variables were reported as mean \pm s.d.; heterogeneous continuous variables were described as medians or percentiles (25th to 75 th). Categorical variables were reported as percentage $(95 \% \mathrm{Cl})$. 
Table 2 Cardiovascular risk stratification: baPWV/CRAE/retinal vascular fractal dimension

\begin{tabular}{|c|c|c|c|c|}
\hline & $\mathrm{N}$ & $\begin{array}{c}\text { baPWV } \\
\text { Mean (s.e.) }\left(\mathrm{cm} \mathrm{s}^{-1}\right)\end{array}$ & $\begin{array}{c}\text { CRAE } \\
\text { Mean (s.e.) }(\mu m)\end{array}$ & $\begin{array}{l}\text { Retinal vascular fractal dimension } \\
\text { Mean (s.e.) }\end{array}$ \\
\hline \multicolumn{5}{|l|}{ Age (years) } \\
\hline Youngest, 30-39 & 356 & $1203.770(14.856)$ & $134.484(0.628)$ & $1.388(0.003)$ \\
\hline Second, 40-49 & 647 & $1280.656(10.878)$ & $134.241(0.460)$ & $1.380(0.002)$ \\
\hline Third, 50-59 & 575 & $1430.568(11.251)$ & $133.718(0.476)$ & $1.366(0.002)$ \\
\hline \multicolumn{5}{|l|}{ Diabetes } \\
\hline Absent & 1897 & $1408.554(6.225)$ & $133.863(0.263)$ & $1.366(0.001)$ \\
\hline Present & 272 & $1530.131(16.801)$ & $133.712(0.709)$ & $1.359(0.003)$ \\
\hline \multicolumn{5}{|l|}{ Hypertension } \\
\hline \multicolumn{5}{|l|}{$S B P(m m ~ H g)$} \\
\hline \multicolumn{5}{|l|}{ Quartiles } \\
\hline First, 80-110 & 539 & 1254.669 (12.153) & $137.151(0.575)$ & $1.382(0.002)$ \\
\hline Second,111-124 & 545 & $1333.984(10.272)$ & $134.515(0.486)$ & $1.369(0.002)$ \\
\hline Third,125-138 & 539 & $1434.264(10.091)$ & $132.600(0.477)$ & $1.362(0.002)$ \\
\hline Fourth,139-224 & 546 & $1654.833(10.661)$ & $131.457(0.504)$ & $1.352(0.002)$ \\
\hline \multicolumn{5}{|l|}{$D B P(m m ~ H g)$} \\
\hline \multicolumn{5}{|l|}{ Quartiles } \\
\hline First,50-70 & 539 & $1301.558(11.900)$ & $136.513(0.522)$ & $1.374(0.002)$ \\
\hline Second,71-78 & 545 & $1366.965(11.426)$ & $134.738(0.501)$ & $1.372(0.002)$ \\
\hline Third,79-86 & 539 & $1442.583(10.924)$ & $132.579(0.479)$ & $1.361(0.002)$ \\
\hline \multicolumn{5}{|l|}{ Quartiles } \\
\hline \multicolumn{5}{|l|}{ Pulse pressure $(\mathrm{mm} \mathrm{Hg})$} \\
\hline \multicolumn{5}{|l|}{ Quartiles } \\
\hline First, 8-38 & 539 & $1316.672(12.184)$ & $135.672(0.545)$ & $1.372(0.002)$ \\
\hline Second, 38-45 & 545 & $1355.064(11.074)$ & $133.846(0.496)$ & $1.370(0.002)$ \\
\hline Third, 46-56 & 539 & $1418.191(10.876)$ & $133.689(0.487)$ & $1.364(0.002)$ \\
\hline Fourth, 56-134 & 546 & $1603.590(11.507)$ & $132.329(0.515)$ & $1.356(0.002)$ \\
\hline \multicolumn{5}{|l|}{$B M I\left(\mathrm{~kg} \mathrm{~m}^{-2}\right)$} \\
\hline Normal or malnutrition, $<25$ & 1473 & $1402.623(6.945)$ & $134.626(0.289)$ & $1.368(0.001)$ \\
\hline Overweight or obesity, $\geqslant 25$ & 696 & 1449.659 (10.157) & $132.992(0.423)$ & $1.360(0.002)$ \\
\hline \multicolumn{5}{|l|}{$L D L-C\left(\mathrm{mmol}^{-1}\right)$} \\
\hline Normal, $<3.3$ & 1692 & $1412.109(6.715)$ & $134.058(0.280)$ & $1.366(0.001)$ \\
\hline Abnormal, $\geqslant 3.3$ & 477 & 1464.543 (12.399) & $133.152(0.518)$ & $1.363(0.002)$ \\
\hline
\end{tabular}

Abbreviations: baPWV, brachial-ankle pulse wave velocity; BMI, body mass index; CRAE, central retinal arteriolar equivalent; DBP, diastolic blood pressure; LDL-C, low density lipoprotein cholesterol; MABP, mean arterial blood pressure; SBP, systolic blood pressure; TC, total cholesterol.

Data are means and s.e., adjusted gender and age if applicable. All tests for trends are significant $(P<0.05)$. 


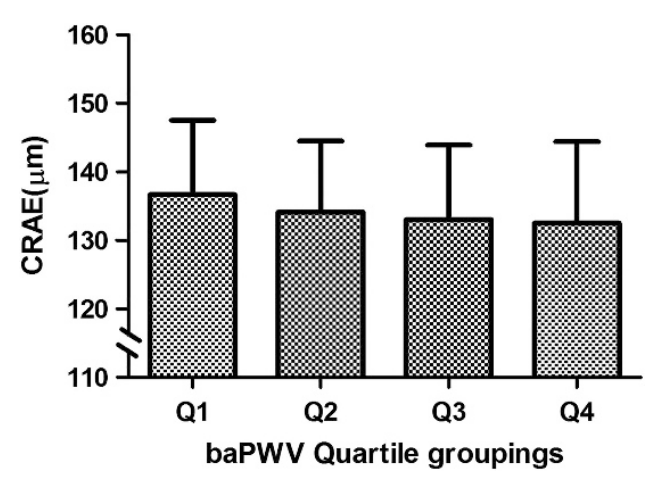

Figure 3 The CRAE distribution in the baPWV quartile groupings.

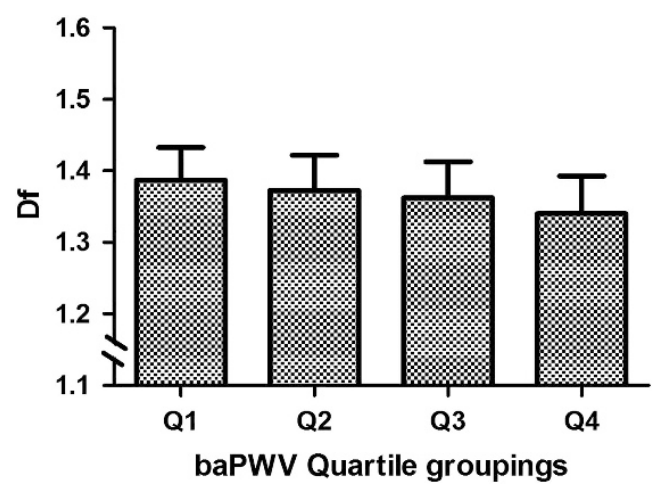

Figure 4 Retinal vascular fractal dimension distribution in the baPWV quartile groupings.

Adjusting for the gender and age, the baPWV gradually increased with the increase in age, systolic blood pressure, diastolic blood pressure, mean arterial blood pressure, pulse pressure, BMI, total cholesterol and LDL-C, whereas the CRAE and retinal vascular fractal dimension were gradually reduced. The baPWVs in the diabetic and hypertensive subjects were higher than those in the non-diabetic or non-hypertensive subjects, whereas the CRAEs and retinal vascular fractal dimensions in the diabetic and hypertensive subjects were lower than those in the non-diabetic or non-hypertensive subjects $(P<0.05)$ (Table 2).

The CRAE and retinal vascular fractal dimension decreased with the increase in the quartile of baPWV (Q1-Q4) $(P<0.05)$ (Figures 3 and 4), and analysis of covariance was statistically significant after the gender and age were adjusted $(P<0.001)$ (Table 3). Defining baPWV as a dependent variable, CRAE and retinal vascular fractal dimension as independent variables, and adjusting for the gender, age, smoking habits and alcohol consumption, logistic regression analysis revealed that the likelihood of the central retinal artery narrowing (odds ratio $=3.093 ; 95 \%$ confidence interval: $2.010-4.760 ; P<0.001)$ and the retinal vascular fractal dimension decreasing (odds ratio $=3.746$; $95 \%$ confidence interval: $2.312-6.070 ; P<0.001$ ) increased with the elevated baPWV (Q1-Q4). Further adjustment of the cardiovascular risk factors diminished the association between baPWV and CRAE, but increased the association between baPWV and the retinal vascular fractal dimension (Table 4).
Table 3 Association of baPWV with CRAE and retinal vascular fractal dimension

\begin{tabular}{cccc}
\hline & & $\begin{array}{c}\text { CRAE }(\mu \mathrm{m}) \\
\text { Mean (s.e.) }\end{array}$ & $\begin{array}{c}\text { Retinal vascular fractal dimension } \\
\text { Mean (s.e.) }\end{array}$ \\
\hline baPWV (cm s & \\
Q1, 770-1169 & 539 & $136.730(0.527)$ & $1.375(0.002)$ \\
Q2,1170-1346 & 546 & $134.290(0.481)$ & $1.367(0.002)$ \\
Q3,1347-1599 & 539 & $133.121(0.479)$ & $1.365(0.002)$ \\
Q4,1600-3746 & 545 & $132.283(0.539)$ & $1.355(0.002)$ \\
& & & $<0.001$ \\
\hline -value & & $<0.001$ & $<$ \\
\hline
\end{tabular}

Abbreviations: baPWV, brachial-ankle pulse wave velocity; CRAE, central retinal arteriolar equivalent.

Data are means and s.e., adjusted gender and age.

aTest for trends.

\section{DISCUSSION}

Our findings showed that the CRAE and the retinal vascular fractal dimension were reduced with an increase in baPWV, independent of gender, age, smoking habits, alcohol consumption, diabetes, hypertension, BMI, abnormal serum lipid level, hs-CRP level and the SUA level. The association of the large- and medium-sized arterial stiffness with the retinal arteriolar diameter and the retinal vascular complexity provides an important perspective from which to evaluate the correlation between the aorta and the arteriole or the microvasculature. The correlation between the arterial stiffness and the retinal microvascular changes has been recently proved in clinical trials. ${ }^{27-30}$ However, the mechanisms underlying the correlation remain unclear. Changes in the structure and the blood flow velocity of the arteriole or the microvascular structures carrying nutrients may affect the aortic function. ${ }^{36-39}$ Atherosclerosis of the large- and medium-sized arteries increases the blood flow to the arteriole with high pulsatility index, resulting in vascular damage. ${ }^{40}$ In addition, the interaction promotes the progression of atherosclerosis. Conversely, the pathology leading to changes in aortic walls and arterioles, including endothelial dysfunction, modified collagen ratio and atherosclerosis, may develop in parallel with the atherosclerosis of the large- and medium-sized arteries.

\section{baPWV and CRAE}

Although the study methods used were different, the correlation between the aortic stiffness and the retinal arteriolar narrowing has been widely investigated. It has been reported that the general retinal arteriolar narrowing correlates with clinical stroke, ${ }^{41}$ impaired cardiac systolic function ${ }^{42}$ and subclinical leukodystrophy. ${ }^{43}$ A study involving black and white subjects showed that increased carotid artery stiffness was associated with decreased arteriole-to-venule ratio in middle-aged people. ${ }^{28}$ In addition, a multiethnic study of atherosclerosis involving 3425 participants from multiple ethnic backgrounds revealed that the increased arterial stiffness was associated with retinal arteriolar narrowing, although such a link was not apparent in Chinese women. ${ }^{29}$

The present study involved the inhabitants from the southeastern coast of China. We found a negative correlation between baPWV and CRAE after the cardiovascular risk factors including systolic blood pressure, diabetes, BMI and abnormal serum lipid level were adjusted, indicating that the baPWV increases with the reduction in CRAE. It suggested that hypertension affects arterial stiffness through altered arterial compliance, elasticity, distensibility and arterial wall 
Table 4 Multiple logistic regression of CRAE/retinal vascular fractal dimension by baPWV

OR $(95 \% \mathrm{Cls})$ CRAE/retinal vascular fractal dimension lowest quartile

\begin{tabular}{|c|c|c|c|c|c|}
\hline \multirow[b]{2}{*}{ baPWV } & & & & & \multirow[b]{2}{*}{ P-value } \\
\hline & Q1 & Q2 & Q3 & Q4 & \\
\hline \multicolumn{6}{|l|}{ CRAE } \\
\hline Model $1^{b}$ & 1.000 (Ref.) & $2.001(1.394,2.872)$ & $2.889(1.967,4.243)$ & $3.093(2.010,4.760)$ & $<0.001$ \\
\hline Model $2^{c}$ & 1.000 (Ref.) & $1.759(1.224,2.528)$ & $2.083(1.408,3.081)$ & $1.635(1.031,2.595)$ & $<0.001$ \\
\hline \multicolumn{6}{|c|}{ Retinal vascular fractal dimension } \\
\hline
\end{tabular}

baPWV, brachial ankle pulse wave velocity; BMI, body mass index; $\mathrm{Cl}$, confidence interval; CRAE, central retinal arteriolar equivalent; hs-CRP, hypersensitive C-reactive protein; OR, odds ratio; Q1Q4, quartiles of baPWV; SUA, serum uric acid.

aTest for trends.

bModel 1: adjusted by gender, age, cigarette smoking and alcohol consumption.

'Model 2: adjusted by diabetes, systolic blood pressure, BMI, dyslipidemia, hs-CRP and SUA.

composition. The vascular stiffness enables more rapid reflux of the peripheral blood, leading to increased central systolic and pulse pressure. ${ }^{43}$ Multiple lines of evidence support the correlation between CRAE and hypertension, pointing to a further link between baPWV and CRAE. ${ }^{2,4-6,44-47}$ The key factor linking baPWV and CRAE may be the increased pulsatility index of the blood flow entering the small blood vessels, which is caused by the huge differences in arterial blood pressure, ${ }^{29}$ or the injury to the arteriole supplying nutrients, as well as structural and functional alterations of the aortic wall. ${ }^{36-38}$

Other studies evaluating the increased ambulatory arterial stiffness index imply a greater presence of subclinical organ damage in primary hypertensive patients. This finding was somewhat consistent with our results that blood pressure may have a part in the relationship between baPWV and CRAE. ${ }^{48}$

\section{baPWV and retinal vascular fractal dimension}

The retinal vascular fractal dimension is a measure of the retinal vascular complexity and is more stable than CRAE. Our results also showed that the correlation between baPWV and the retinal vascular fractal dimension may be stronger than that between baPWV and CRAE. It has been shown that the retinal vascular fractal dimension measurement was not affected by the resolution of the fundus photograph and was hardly affected by the vascular pulsation. In addition, it was not affected by the number of regional blood vessels even if the measurement was based on the vascular bifurcation, the bifurcation angle and the length of the blood vessel between the bifurcations. ${ }^{9}$ It was suggested that the retinal vascular fractal dimension should be used to reflect the occurrence, severity and target organ injury in hypertensive patients and the patient response to antihypertensive drugs. ${ }^{49-51}$ Similar to CRAE, the retinal vascular fractal dimension is a predictor of cardiovascular disease. ${ }^{52}$ Based on the evidence available so far, it is considered that an increased baPWV and a reduced retinal vascular fractal dimension are independently observed in hypertensive subjects, ${ }^{9}$ and a stronger correlation between baPWV and the retinal vascular fractal dimension was observed in hypertensive subjects than in non-hypertensive subjects. Hypertension reportedly promotes vascular remodeling and sparse distribution of microblood vessels, and the arteriolar narrowing in hypertension causes an increased resistance to distal blood flow and compensatory sparse distribution of the microvascular network that is characterized by reduced retinal vascular fractal dimension. ${ }^{49}$
To our knowledge, this is the first study to evaluate the association between the quantitative parameters of the retinal blood vessels and aortic stiffness in Chinese inhabitants. The present study involved a series of cardiovascular risk factors including SUA, and used noninvasive baPWV to describe aortic stiffness, which facilitated the enrollment of additional study subjects. However, the current study has the following limitations: (1) this study is not a prospective study and cannot identify the causal relationship between the retinal vascular parameters and aortic stiffness; (2) a few subjects were not enrolled in the study because of noncompliance, leading to possible selection bias; and (3) we did not use the combination of carotid-femoral pulse wave velocity, carotid artery intima-media thickness and plain MRI scanning of the aorta to reflect the systemic atherosclerotic changes.

\section{Conclusion}

This is the first study to evaluate the association between the quantitative parameters of the retinal blood vessels and aortic stiffness in Chinese inhabitants. The study indicates that the CRAE and the retinal vascular fractal dimension were reduced with an increase in baPWV. The association of the large- and medium-sized arterial stiffness with the retinal arteriolar diameter and the retinal vascular complexity provides an important perspective to evaluate the correlation between the aorta and the arterioles or microvasculature. Similar to CRAE, the retinal vascular fractal dimension is a predictor of cardiovascular diseases. The current study revealed that the correlation between baPWV and the retinal vascular fractal dimension was significantly stronger in hypertensive subjects than in nonhypertensive subjects

\section{CONFLICT OF INTEREST}

The authors declare no conflict of interest.

\section{ACKNOWLEDGEMENTS}

Our study was supported by the Fujian Major Program of Basic Science Project Foundation via projects entitled 'Epidemiologic survey of hypertension and prehypertension intervention research in HaiDao County of Fujian Province' (2010Y0013), 'Predictive value of quantitative parameters of retinal vessel for subclinical atherosclerosis and their relationship with other cardiovascular risk factors' (2013Y0023) and the Fujian Medical Innovation Project entitled 'The association of plasma apelin levels and apelin polymorphism with hypertension and hypertensive vascular injury' (2012-CX-1). 
1 Hubbard LD, Brothers RJ, King WN, Clegg LX, Klein R, Cooper LS, Sharrett AR, Davis MD, Cai J. Methods for evaluation of retinal microvascular abnormalities associated with hypertension/sclerosis in the Atherosclerosis Risk in Communities Study. Ophthalmology 1999; 106: 2269-2280.

2 Wong TY, Klein R, Sharrett AR, Duncan BB, Couper DJ, Tielsch JM, Klein BE, Hubbard LD. Retinal arteriolar narrowing and risk of coronary heart disease in men and women. The Atherosclerosis Risk in Communities Study. JAMA 2002; 287 1153-1159.

3 Ikram MK, de Jong FJ, Vingerling JR, Witteman JC, Hofman A, Breteler MM, de Jong PT Are retinal arteriolar or venular diameters associated with markers for cardiovascular disorders? The Rotterdam Study. Invest Ophthalmol Vis Sci 2004; 45: 2129-2134.

4 Ikram MK, de Jong FJ, Bos MJ, Vingerling JR, Hofman A, Koudstaal PJ, de Jong PT, Breteler MM. Retinal vessel diameters and risk of stroke: the Rotterdam Study. Neurology 2006; 66: 1339-1343.

5 Wong TY, Kamineni A, Klein R, Sharrett AR, Klein BE, Siscovick DS, Cushman M, Duncan BB. Quantitative retinal venular caliber and risk of cardiovascular disease in older persons: the cardiovascular health study. Arch Intern Med 2006; 166: 2388-2394.

6 Wong TY, Islam FM, Klein R, Klein BE, Cotch MF, Castro C, Sharrett AR, Shahar E. Retinal vascular caliber, cardiovascular risk factors, and inflammation: the multi-ethnic study of atherosclerosis (MESA). Invest Ophthalmol Vis Sci 2006; 47: 2341-2350.

7 Sherman TF. On connecting large vessels to small. The meaning of Murray's law. J Gen Physiol 1981; 78: 431-453.

8 Cheung CY, Tay WT, Mitchell P, Wang JJ, Hsu W, Lee ML, Lau QP, Zhu AL, Klein R, Saw SM, Wong TY. Quantitative and qualitative retinal microvascular characteristics and blood pressure. J Hypertens 2011; 29: 1380-1391.

9 Liew G, Wang JJ, Cheung N, Zhang YP, Hsu W, Lee ML, Mitchell P, Tikellis G, Taylor B, Wong TY. The retinal vasculature as a fractal: methodology, reliability, and relationship to blood pressure. Ophthalmology 2008; 115: 1951-1956.

10 Lim SW, Cheung N, Wang JJ, Donaghue KC, Liew G, Islam FM, Jenkins AJ, Wong TY Retinal vascular fractal dimension and risk of early diabetic retinopathy: a prospective study of children and adolescents with type 1 diabetes. Diabetes Care 2009; 32 2081-2083.

11 Cheung N, Donaghue KC, Liew G, Rogers SL, Wang JJ, Lim SW, Jenkins AJ, Hsu W, Li Lee M, Wong TY. Quantitative assessment of early diabetic retinopathy using fractal analysis. Diabetes Care 2009; 32: 106-110.

12 Sng CC, Sabanayagam C, Lamoureux EL, Liu E, Lim SC, Hamzah H, Lee J, Tai ES, Wong TY. Fractal analysis of the retinal vasculature and chronic kidney disease. Nephrol Dial Transplant 2010; 25: 2252-2258.

13 Cheung N, Liew G, Lindley RI, Liu EY, Wang JJ, Hand P, Baker M, Mitchell P, Wong TY, Multi-Center R, Stroke Study Collaborative G. Retinal fractals and acute lacunar stroke. Ann Neurol 2010; 68: 107-111.

14 Kawasaki R, Che Azemin MZ, Kumar DK, Tan AG, Liew G, Wong TY, Mitchell P, Wang JJ. Fractal dimension of the retinal vasculature and risk of stroke: a nested casecontrol study. Neurology 2011; 76: 1766-1767.

15 Doubal FN, MacGillivray TJ, Patton N, Dhillon B, Dennis MS, Wardlaw JM. Fractal analysis of retinal vessels suggests that a distinct vasculopathy causes lacunar stroke. Neurology 2010; 74: 1102-1107.

16 Wang S, Xu L, Wang Y, Wang Y, Jonas JB. Retinal vessel diameter in normal and glaucomatous eyes: the Beijing eye study. Clin Exp Ophthalmol 2007; 35: 800-807.

17 Laurent S, Katsahian S, Fassot C, Tropeano Al, Gautier I, Laloux B, Boutouyrie P. Aortic stiffness is an independent predictor of fatal stroke in essential hypertension. Stroke 2003; 34: 1203-1206.

18 Hodes RJ, Lakatta EG, McNeil CT. Another modifiable risk factor for cardiovascular disease? Some evidence points to arterial stiffness. J Am Geriatr Soc 1995; 43 581-582.

19 Arnett DK, Boland LL, Evans GW, Riley W, Barnes R, Tyroler HA, Heiss G. Hypertension and arterial stiffness: the Atherosclerosis Risk in Communities Study. ARIC Investigators. Am J Hypertens 2000; 13: 317-323.

20 Cockcroft JR, Webb DJ, Wilkinson IB. Arterial stiffness, hypertension and diabetes mellitus. J Hum Hypertens 2000; 14: 377-380.

21 Stefanadis C, Tsiamis E, Vlachopoulos C, Stratos C, Toutouzas K, Pitsavos C, Marakas S, Boudoulas H, Toutouzas P. Unfavorable effect of smoking on the elastic properties of the human aorta. Circulation 1997; 95: 31-38.

22 Laurent S, Boutouyrie P, Asmar R, Gautier I, Laloux B, Guize L, Ducimetiere P, Benetos A. Aortic stiffness is an independent predictor of all-cause and cardiovascular mortality in hypertensive patients. Hypertension 2001; 37: 1236-1241.

23 Blacher J, Asmar R, Djane S, London GM, Safar ME. Aortic pulse wave velocity as a marker of cardiovascular risk in hypertensive patients. Hypertension 1999; 33 1111-1117.

24 Asmar R, Rudnichi A, Blacher J, London GM, Safar ME. Pulse pressure and aortic pulse wave are markers of cardiovascular risk in hypertensive populations. Am J Hypertens 2001; 14: 91-97.

25 Meaume S, Rudnichi A, Lynch A, Bussy C, Sebban C, Benetos A, Safar ME. Aortic pulse wave velocity as a marker of cardiovascular disease in subjects over 70 years old J Hypertens 2001; 19: 871-877.

26 Vlachopoulos C, Aznaouridis K, Stefanadis C. Prediction of cardiovascular events and all-cause mortality with arterial stiffness: a systematic review and meta-analysis. J Am Coll Cardiol 2010; 55: 1318-1327.

27 Cheung N, Islam FM, Jacobs DR Jr, Sharrett AR, Klein R, Polak JF, Cotch MF, Klein BE, Ouyang P, Wong TY. Arterial compliance and retinal vascular caliber in cerebrovascula disease. Ann Neurol 2007; 62: 618-624.
28 Liao D, Wong TY, Klein R, Jones D, Hubbard L, Sharrett AR. Relationship between carotid artery stiffness and retinal arteriolar narrowing in healthy middle-aged persons. Stroke 2004; 35: 837-842.

29 Cheung N, Sharrett AR, Klein R, Criqui MH, Islam FM, Macura KJ, Cotch MF, Klein BE, Wong TY. Aortic distensibility and retinal arteriolar narrowing: the multi-ethnic study of atherosclerosis. Hypertension 2007; 50: 617-622.

30 De Silva DA, Woon FP, Manzano JJ, Liu EY, Chang HM, Chen C, Wang JJ, Mitchell P, Kingwell BA, Cameron JD, Lindley RI, Wong TY, Wong MC. on behalf of the MultiCentre Retinal Stroke Study Collaborative G. The relationship between aortic stiffness and changes in retinal microvessels among Asian ischemic stroke patients. J Hum Hypertens 2012; 26: 716-722.

31 Knudtson MD, Lee KE, Hubbard LD, Wong TY, Klein R, Klein BE. Revised formulas for summarizing retinal vessel diameters. Curr Eye Res 2003; 27: 143-149.

32 Chobanian AV, Bakris GL, Black HR, Cushman WC, Green LA, Izzo JL Jr, Jones DW, Materson BJ, Oparil S, Wright JT Jr, Roccella EJ. National Heart L. The Seventh Report of the Joint National Committee on Prevention, Detection, Evaluation, and Treatment of High Blood Pressure: the JNC 7 report. JAMA 2003; 289 2560-2572.

33 American Diabetes Association. Standards of medical care in diabetes-2011. Diabetes Care 2011; 34: S11-S61.

34 Yamashina A, Tomiyama H, Arai T, Hirose K, Koji Y, Hirayama Y, Yamamoto Y, Hori S Brachial-ankle pulse wave velocity as a marker of atherosclerotic vascular damage and cardiovascular risk. Hypertens Res 2003; 26: 615-622.

35 Sabanayagam C, Shankar A, Klein BE, Lee KE, Muntner P, Nieto FJ, Tsai MY, Cruickshanks KJ, Schubert CR, Brazy PC, Coresh J, Klein R. Bidirectional association of retinal vessel diameters and estimated glomerular filtration rate decline: The Beaver Dam CKD Study. Am J Kidney Dis 2011; 57: 682-691.

36 Stefanadis Cl, Karayannacos PE, Boudoulas HK, Stratos CG, Vlachopoulos CV, Dontas IA, Toutouzas PK. Medial necrosis and acute alterations in aortic distensibility following removal of the vasa vasorum of canine ascending aorta. Cardiovasc Res 1993 27: 951-956

37 Angouras D, Sokolis DP, Dosios T, Kostomitsopoulos N, Boudoulas H, Skalkeas G, Karayannacos PE. Effect of impaired vasa vasorum flow on the structure and mechanics of the thoracic aorta: implications for the pathogenesis of aortic dissection. Eur $J$ Cardiothorac Surg 2000; 17: 468-473.

38 Stefanadis C, Vlachopoulos C, Tsiamis E, Diamantopoulos L, Toutouzas K, Giatrakos N, Vaina S, Tsekoura D, Toutouzas P. Unfavorable effects of passive smoking on aortic function in men. Ann Intern Med 1998; 128: 426-434.

39 Zagura M, Kals J, Serg M, Kampus P, Zilmer M, Jakobson M, Unt E, Lieberg J, Eha J. Structural and biochemical characteristics of arterial stiffness in patients with atherosclerosis and in healthy subjects. Hypertens Res 2012; 35 1032-1037.

40 Vlachopoulos C, Dima I, Aznaouridis K, Vasiliadou C, loakeimidis N, Aggeli C, Toutouza M, Stefanadis $C$. Acute systemic inflammation increases arterial stiffness and decreases wave reflections in healthy individuals. Circulation 2005; 112: 2193-2200.

41 Wong TY, Klein R, Couper DJ, Cooper LS, Shahar E, Hubbard LD, Wofford MR, Sharrett AR. Retinal microvascular abnormalities and incident stroke: the Atherosclerosis Risk in Communities Study. Lancet 2001; 358: 1134-1140.

42 Wong TY, Klein R, Sharrett AR, Nieto FJ, Boland LL, Couper DJ, Mosley TH, Klein BE, Hubbard LD, Szklo M. Retinal microvascular abnormalities and cognitive impairment in middle-aged persons: the Atherosclerosis Risk in Communities Study. Stroke 2002; 33 1487-1492.

43 Feihl F, Liaudet L, Waeber B. The macrocirculation and microcirculation of hypertension. Curr Hypertens Rep 2009; 11: 182-189.

44 Sun C, Liew G, Wang JJ, Mitchell P, Saw SM, Aung T, Tai ES, Wong TY. Retinal vascular caliber, blood pressure, and cardiovascular risk factors in an Asian population the Singapore Malay Eye Study. Invest Ophthalmol Vis Sci 2008; 49: 1784-1790.

45 Kawasaki R, Wang JJ, Rochtchina E, Taylor B, Wong TY, Tominaga M, Kato T, Daimon M, Oizumi T, Kawata S, Kayama T, Yamashita H, Mitchell P. Cardiovascular risk factors and retinal microvascular signs in an adult Japanese population: the Funagata Study Ophthalmology 2006; 113: 1378-1384.

46 Smith W, Wang JJ, Wong TY, Rochtchina E, Klein R, Leeder SR, Mitchell P. Retinal arteriolar narrowing is associated with 5-year incident severe hypertension: the Blue Mountains Eye Study. Hypertension 2004; 44: 442-447.

47 Wang JJ, Liew G, Wong TY, Smith W, Klein R, Leeder SR, Mitchell P. Retinal vascula calibre and the risk of coronary heart disease-related death. Heart 2006; 92 1583-1587.

48 García-García A, Gómez-Marcos MA, Recio-Rodriguez JI, González-Elena LJ, ParraSanchez J, Fe Muñoz-Moreno M, Alonso CP, Gude F, García-Ortiz L. Relationship between ambulatory arterial stiffness index and subclinical target organ damage in hypertensive patients. Hypertens Res 2011; 34: 180-186.

49 Daxer A. Characterisation of the neovascularisation process in diabetic retinopathy by means of fractal geometry: diagnostic implications. Graefes Arch Clin Exp Ophthalmol 1993; 231: 681-686.

50 Daxer A. The fractal geometry of proliferative diabetic retinopathy: implications for the diagnosis and the process of retinal vasculogenesis. Curr Eye Res 1993; 12: 1103-1109.

51 Avakian A, Kalina RE, Sage EH, Rambhia AH, Elliott KE, Chuang EL, Clark JI, Hwang $J N$, Parsons-Wingerter P. Fractal analysis of region-based vascular change in the normal and non-proliferative diabetic retina. Curr Eye Res 2002; 24: 274-280.

52 Wong TY, Mitchell P. The eye in hypertension. Lancet 2007; 369: 425-435. 